\title{
Cost-effectiveness of palbociclib plus letrozole versus letrozole alone as a first-line treatment in women with oestrogen receptor- positive, HER2-negative, advanced breast cancer. Revised results for the Swiss health care setting
}

\author{
K. Matter-Walstra ${ }^{1,2}$ (D) M. Schwenkglenks ${ }^{1} \cdot$ K. J. Dedes ${ }^{2,3}$
}

Received: 14 March 2017 / Accepted: 15 March 2017/Published online: 21 March 2017

(C) Springer Science+Business Media New York 2017

In 2016, we published a cost-effectiveness analysis of palbociclib plus letrozole versus letrozole alone as a firstline treatment in women with oestrogen receptor-positive, HER2-negative, advanced breast cancer, for the Swiss health care setting [1]. For this analysis, we used the US price of palbociclib, the only price available at the time. The US price was converted to Swiss Francs, resulting in a price per mg of CHF 3.69. This led to a base case incremental cost-effectiveness ratio (ICER) of the combination therapy of CHF 301,227 per QALY gained. In March 2017, palbociclib was approved for reimbursement by the Swiss statutory health insurance in combination with fulvestrant. The set Swiss price for palbociclib is substantially lower than we assumed in our original analysis. A package of 21 tablets of $125 \mathrm{mg}$ (https://compendium.ch/search/palboci clib/en) costs CHF 4145.50, resulting in a price per mg of CHF 1.58. We recalculated the ICER with this new price. In the base case (no discounting due to short life expectancy of the patient population studied), the ICER is now CHF 137,063 per QALY gained, 55\% lower than the initially calculated ICER. When using discount rates of 3 and $6 \%$, the ICER increases to CHF 143,585 and CHF 150,341 per QALY gained, respectively. Compared to an assumed willingness to pay threshold of CHF 100,000 per QALY gained, the probability that palbociclib plus letrozole compared to letrozole alone is cost effective is now $19 \%$ in the base case. Based on 2008-2012 data, mortality from

K. Matter-Walstra

klazien.matter@unibas.ch

University Basel, Basel, Switzerland

2 Swiss Group of Clinical Cancer Research (SAKK) Coordinating Centre, Bern, Switzerland

3 University Hospital Zürich, 8091 Zurich, Switzerland breast cancer in Switzerland was 21 per 100,000 women, implying approximately 650 breast cancer deaths per year in a female population of 3,956,781 (www.bfs.admin.ch). If $78 \%$ [2] of the patients' cancers were oestrogen receptor-positive, HER2-negative, and assuming that $70 \%$ would be treated with palbociclib plus letrozole, the yearly budget impact for the Swiss health care system might be in the range of CHF143 million, approximately CHF 70 million higher than for treatment with letrozole alone. Determination of the health economic characteristics of palbociclib in combination with fulvestrant, the combination now approved for reimbursement by the Swiss statutory health insurance, would require a separate health economic study.

Acknowledgements We thank the SAKK Coordinating Centre for reviewing our manuscript.

Funding The Swiss State Secretariat for Education, Research and Innovation (SERI) supported the original work.

Compliance with ethical standards

Conflict of interest All authors declared no potential conflicts of interest related to palbociclib or other drugs mentioned in this article. Authors have no financial relationship with the organization that sponsored the research.

\section{References}

1. Matter-Walstra K, Ruhstaller T, Klingbiel D et al (2016) Palbociclib as a first-line treatment in oestrogen receptor-positive, HER2-negative, advanced breast cancer not cost-effective with current pricing: a health economic analysis of the Swiss Group for Clinical Cancer Research (SAKK). Breast Cancer Res Treat 158:51-57

2. Diebold J, Aebi S (2014) Prognostische und prädiktive Marker beim Mammakarzinom. SZO 1:10-14 